\section{Origin of Chromosome Linkage in Enothera.}

Several years ago the hypothesis was put forward that in Enothera new species have arisen through crossing and been perpetuated by the chromosome linkage which, it was assumed, had arisen as a result of crossing between unrelated species (Gates, 1928). ${ }^{1}$ It has recently been possible to test this hypothesis experimentally, with results which show that chromosome linkage (catenation) ${ }^{2}$ can arise when species or mutants showing no catenation are crossed together.

It is known from previous work that $C E$. deserens and $C E$. blandina, both of which are secondary mutant derivatives from $E$. Lamarckiana, have seven free pairs of chromosomes in meiosis, and that the same condition exists in $C E$. purpurata, a species described from Germany by Klebahn. ${ }^{3}$ Reciprocal crosses were made between these three forms, which thus furnish us with a triangle of species in which the bivalent chromosomes were all unlinked in meiosis.

A preliminary examination of some of the $F_{1}$ hybrids shows the following catenations : deserens $x$ blandina, ring of 6 and 4 pairs ; deserens $\times$ purpurata, ring of 4 and 5 pairs; blandina $\times$ purpurata, ring of 4 and 5 pairs. In all three crosses catenation has thus arisen through hybridisation between forms having only paired chromosomes.

The view is thus experimentally confirmed that the species of Enothera, most of which show catenation, have arisen through crossing between previous species and breed true because of the genetic linkage resulting from that catenation. That heterozygous species can breed true because of apogamous reproduction is a familiar conception. That they can breed true because of catenation (linkage) of the chromosomes in meiosis is not generally recognised, but is now well known in CEnothera.

Without going into details, it seems clear that the chromosome-complexes of deserens and blandina have become different by two chromosome segmental interchanges, involving three non-homologous chromosomes. The results of crossing these forms with EE. purpurata may be explained on the assumption that purpurata differs from blandina by one of these segmental exchanges and from deserens by the other.

A full account of this work and its bearing on problems of chromosome linkage will be published later.

R. Ruggles Gates.

King's College, London, D. G. Catcheside. Sept. 22

1 Zeit.für Abst. u. Vererb. Suppt. Vol., p. 752. Bibliographia Genetica, vol. 4 , p. 480

vol. 65 , p. $97 ; 1931$

3 See Gates and Goodwin, Proc. Roy. Soc., B, in press.

\section{Magnetic Resolution and Nuclear Moment of Rhenium.}

For some time the spectrum of rhenium has been the subject of investigation in the Amsterdam Laboratory " Physica". With the Hilger $\mathrm{E}_{1}$ quartz spectrograph we have studied the spectrum of the arc and of the underwater spark. The hyperfine structure and the magnetic resolution have been investigated with the 20-ft. grating in an Eagle mounting.

The following strong complex arc lines: $\lambda \lambda 3465$; 3460 ; 3452 ; 3424 ; 3399 A., which give absorption in the underwater spark and therefore should be considered as combinations with the ground-level $\left(d^{5} s^{2}{ }^{6} S_{5} / 2\right)$, are of special interest. Meggers ${ }^{1}$ has identified the first three lines as the combinations ${ }^{6} S_{5 / 2}-{ }^{6} P_{5} / 27 / 2{ }^{7} / 2$.

From the number of satellites and from the interval rule we conclude that the hyperfine structure originates from the $d^{5} s p^{6} P$-term, and that the nuclear moment of rhenium is $\frac{5}{2}$, as was suspected by Gremmer and Ritschl.2

The hyperfine structure in the Zeeman components of the line $\lambda 3460{ }^{6} S_{5 / 2}-{ }^{6} P_{1} / 2$ has been resolved. The strongest $\sigma$ component is split up into six nearly equidistant hyperfine structure components, which indicate also that the nuclear moment is $\frac{5}{2}$. The mean distance of these components is $0.334 \mathrm{~cm} .^{-1}$ and gives an interval constant $a$ of $0.095 \mathrm{~cm}^{-1}$ in accordance with the value $0.096 \mathrm{~cm}^{-1}$ obtained from the hyperfine structure without magnetic field. On these six components are partly superposed more feeble groups of non-resolved $\sigma$-components.

It seems that the ${ }^{6} P$-term has a slightly anomalous $g$-value, 1.76 (normal value, 1.714). The observed values for the six subcomponents of the strongest $\sigma$-components and the values calculated with $g=1.76$ and the interval constant of the hyperfine structure without magnetic field are given below. Magnetic field : 38,900 gauss.

$\begin{array}{llllll}\text { Obs. }+2.91 & +2.62 & +2.24 & +1.95 & +1.60 & +1.25 \\ \text { Calc. }+2.94 & +2.60 & +2.26 & +1.93 & +1.59 & +1.26 \\ \text { Obs. }-2.90 & -2.60 & -2.28 & -1.93 & -1.61 & -1.24 \\ \text { Calc. }-2.94 & -2.60 & -2.26 & -1.93 & -1.59 & -1.26\end{array}$

We are expecting to make a further report as soon as the investigation can be completed.

\section{P. Zeeman. \\ J. H. Gisolf.}

Laboratory " Physica ",

T. L. DE BRUIN.

University of Amsterdam, Sept. 16.

1 Meggers, Phys. Rev., 37, 219 ; 1931.

Gremmer und Ritschl: Zeit. für Instrumentenkunde, 51, 170; 1981

\section{Infra-Red Absorption Spectrum of Carbonyl Sulphide.}

RECENT developments in the theory of band spectra in the infra-red ${ }^{1}$ lend particular interest to all new experimental results for triatomic molecules. We have recently examined the absorption spectrum of carbonyl sulphide, making use of a monochromator method in order to avoid the photochemical decomposition to be expected on exposure of this substance to the full energy of a Nernst filament. We find a complicated spectrum-a fact which in itself demonstrates the lack of symmetry in the molecule: the results are summarised in Fig. 1. In the few cases in which the bands have been resolved, the separation between the $P$ and $R$ branches is given.

In assigning observed bands to certain fundamental frequencies with their corresponding overtones and combinations, due consideration has to be paid to their relative intensities, which are dependent upon the magnitude and orientation of the effective electric doublets concerned. The latter will be governed by the configuration of the molecule; when this is known, the deduced moments of inertia, the interatomic angles and separations must agree with those obtained by other physical methods, such as X-ray analysis or electron diffraction. Finally, the molecular structure must tally with known chemical and thermochemical data.

We have explored at some length the mechanical possibilities of a system of three different masses having various force constants with $(a)$ covalent and (b) ionic linkings ; the tedious calculations involved have at least enabled us to discard certain forms, but we believe we are justified in directing attention to this particular piece of work, inasmuch as it illustrates the need for cautious statements on such matters.

No. 3232, VoL. 128] 